\title{
Structure and relaxation dynamics of polymer knots
}

\author{
Pik-Yin Lai ${ }^{\mathrm{a}, *}$, Yu-Jane Sheng ${ }^{\mathrm{b}}$, Heng-Kwong Tsao ${ }^{\mathrm{c}}$ \\ ${ }^{a}$ Department of Physics and Center for Complex Systems, National Central University, \\ Chung-li 320, Taiwan, ROC \\ ${ }^{\mathrm{b}}$ Department of Chemical Engineering, National Taiwan University, Taipei 106, Taiwan, ROC \\ ${ }^{c}$ Department of Chemical Engineering, National Central University, Chung-li 320, Taiwan, ROC
}

\begin{abstract}
Monte Carlo simulations are performed to study the equilibrium structure and nonequilibrium dynamic relaxation processes of knotted polymers. We find that topological complexity affects the static and dynamic behavior of knots in different ways to different extent. For statics, our results on the radii of gyration of knot polymers suggest that prime and two-factor composite knots belong to different groups, and we confirm that for knots in the same group, the average radius of gyration scales as $R_{g} \sim N^{3 / 5} p^{-4 / 15}$ in good solvents, where $N$ is the number of monomers and $p$ is the topological invariant representing the length-to-diameter ratio of the knot at its maximum inflated state. From the studies of nonequilibrium relaxation dynamics on prime knots cut at $t=0$, we find that even prime knots should be classified into different groups as $\left(3_{1}, 5_{1}, 7_{1}, \ldots\right),\left(4_{1}, 6_{1}, 8_{1}, \ldots\right),\left(5_{2}, 7_{2}, 9_{2}, \ldots\right)$, etc., based on their topological similarity and their polynomial invariants such as Alexander polynomials. Our results suggest that the mathematical classification of knots can further be parametrized naturally into groups in a way that can have direct physical meaning in terms of structures and dynamics of knots. Furthermore, by scaling calculations, the nonequilibrium relaxation time is found to increase roughly as $p^{12 / 5}$. This prediction is further supported by our data. (C) 2000 Elsevier Science B.V. All rights reserved.
\end{abstract}

PACS: 61.41.+e; 83.10.Nn; 87.10.+e

Keywords: Polymer dynamics; Topological interactions; Knot classification

\section{Introduction}

The physical properties of many biological molecules such as DNAs, are strongly affected by their topological properties which play crucial roles in many

\footnotetext{
* Corresponding author.

E-mail address: pylai@spl1.phy.ncu.edu.tw (P.-Y. Lai)
} 
molecular-biological phenomena. Certain types of topoisomerase enzymes [1-3] which act on circular DNAs, produce different types of DNA knots. Proteins, RNAs and DNAs are all polymers of biological origin. Many molecular biological phenomena are associated with quite ordinary properties and characteristics of polymers. For example, substances like cellulose, polysaccharides, etc., are studied both in the conventional chemistry and in polymer physics together with comparable synthetic substances. Thus, investigations of knotted polymers can be of great help in understanding the behavior of the ring DNAs. Furthermore, recent advances in experimental techniques, such as optical tweezer and fluorescence microscopy can artificially tie up DNA molecules to form a knot structure [4], thus opening the possibility of studying the physical properties of knotted molecules experimentally.

Despite the breakthrough in classifying knots and topological invariants [5,6] and the association of polynomial invariants with many physical spin models [7] and quantum field theory $[8,9]$ in the last decades, the studies on the properties of physical knotted molecules are limited. There have been some studies concentrated on relating the topological invariants of knots to the static properties of the knotted polymers such as developing phenomenological models [10] of the effects of knot complexity on the static and dynamic properties in terms of the number of essential crossings $C$. However, the ability of the theory to predict the equilibrium relaxation dynamics seems questionable. Grosberg et al. [11] presented a mean field theory of the effect of knots on the equilibrium size of ring polymers. In their theory, a topological invariant was introduced which is related to the primitive path in the "polymer in the lattice of obstacles" model. These two works have consistent expression for the effects of topological complexity on the variation of static chain conformations. They are also consistent with the experimental mobility test of DNA knots [12]. It was found that more complex molecules migrate faster than less complex ones which indicates that more complex molecules have more compact conformations. On the other hand, topological interaction manifests itself most prominently in dynamic phenomena in systems of entangled polymer coils or knotted ring polymers. The prohibition against chain crossing in a system restricts the number of its possible conformations. The only conformations are those that are topologically equivalent to one another as permitted by Reidemeister moves [6]. These possible conformations can only appear or disappear via continuous chain deformations without chain crossing nor breaking of the chain connection. Ring polymers which possess topological memory will strongly hinder their relaxation motions. From these aspects, the systems of entangled polymer melts and knotted polymers are somewhat similar in their relaxation behavior.

In this study, not only the equilibrium structure of various types of polymer knots are investigated by Monte Carlo simulations, but also the nonequilibrium dynamic relaxation (untying) process of a knotted polymer cut at one point is studied. Experiments had shown $[1,3]$ that a certain link in a ring DNA breaks up upon the action of topoisomerase and reconnects again after exchanging interlinked strands resulting in a knot structure. The relaxation dynamics of the knotted polymer is therefore important in such process. If the relaxation time is too fast, the knot will untie itself before 
the link reconnects again and no knot structure will result. These results can be used to formulate theoretical models or to be compared with experiments. Our interest is focused on the system in which a ring polymer with a certain topological complexity is initially well equilibrated and then cut at a randomly picked link. The relaxation processes are monitored and analyzed.

\section{Model and simulation details}

Bead spring off-lattice model is employed in the simulation. $N$ beads are connected by stiff springs with interactions between the nonbonded beads through the square-well potential

$$
U_{\mathrm{nb}}= \begin{cases}\infty & (r<\sigma), \\ -\varepsilon & (\sigma \leqslant r<\lambda \sigma), \\ 0 & (\lambda \sigma \leqslant r),\end{cases}
$$

where $\varepsilon$ and $\sigma$ are the energy and size parameters, respectively, and $\lambda=1.5$. The monomeric $\varepsilon$ and $\sigma$ are units used for the reduced quantities for temperature $\left(T^{*}=\right.$ $\left.k_{B} T / \varepsilon\right)$ and distances. The interactions between bonded beads are represented by a cut-off harmonic spring potential as

$$
U_{b}=\frac{1}{2} k \sigma^{2}\left(\frac{r}{\sigma}-1.2\right)^{2}, \quad 1.0<\frac{r}{\sigma} \leqslant 1.4
$$

and the potential is infinite elsewhere. The parameters in the model are chosen to forbid any bond crossing to occur within the knotted chains. $k \sigma^{2} / \varepsilon=400$ and $T^{*}=10$ are chosen so that the system is in the good solvent regime. The system contains a single polymer chain with chain length $N$ ranging from 42 to 82 . We have studied the knotted polymers up to nine crossings: $3_{1}, 4_{1}, 5_{1}, 5_{2}, 6_{1}, 6_{2}, 6_{3}, 7_{1}, 7_{2}, 8_{1}, 9_{1}$, and some composite knots. The standard notation [6] for uniquely labeling a knot is $C_{K}$ where $C$ is the number of essential crossings and $K$ is an index for a particular knot. Fig. 1 displays some of the knot types studied in this work. The simulations are performed under the conditions of constant temperature, volume and total number of beads. The initial configurations are generated by growing the chain bead by bead to the desired length and knot type. The trial moves employed for chains are bead displacement motions [13] which involve randomly picking a bead and displacing it to a new position in the vicinity of the old position. The distance away from the original position is chosen with probability that the condition of equal sampling of all points in the spherical shell surrounding the initial position must be satisfied. The new configurations resulting from this move are accepted according to the standard Metropolis acceptance criterion [14]. All runs are equilibrated for several million steps. Measurements for static properties such as radius of gyration are taken over a period of 1-4 millions MCS/monomer. 

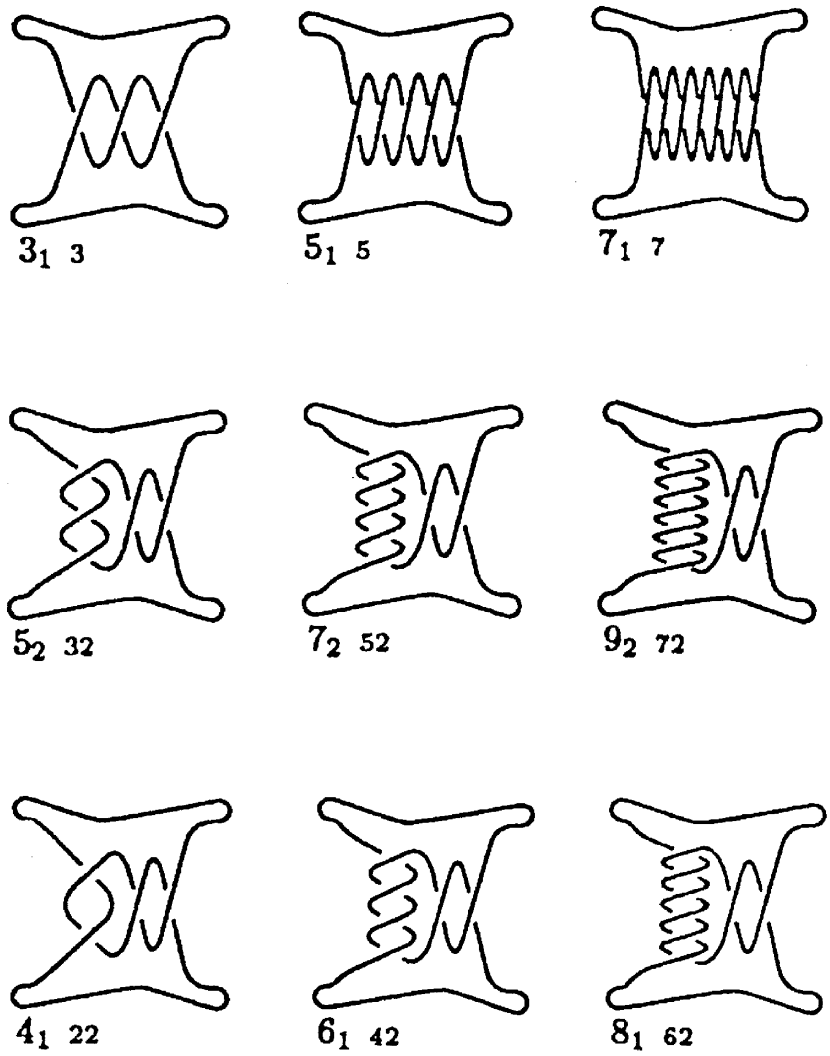

Fig. 1. Schematic knot diagrams. The $\left(3_{1}, 5_{1}, \ldots\right)$ group, the $\left(4_{1}, 6_{1}, \ldots\right)$ group and the $\left(5_{2}, 7_{2}, \ldots\right)$ group. The Conway notation of each knot is also displayed. The first integer in the Conway notation is the number of crossings in the braid structure while the second integer is the number of crossing in the right part of the knot diagram.

The mean radius of gyration

$$
\left\langle R_{g}\right\rangle=\left\langle\sqrt{\frac{1}{N} \sum_{i=1}^{N}\left[\left(x_{i}-x_{\mathrm{cm}}\right)^{2}+\left(y_{i}-y_{\mathrm{cm}}\right)^{2}+\left(z_{i}-z_{\mathrm{cm}}\right)^{2}\right]}\right\rangle
$$

is calculated, where $\left(x_{i}, y_{i}, z_{i}\right)$ are the coordinates of the $i$ th monomer in the chain and $\left(x_{\mathrm{cm}}, y_{\mathrm{cm}}, z_{\mathrm{cm}}\right)$ are the coordinates of the center of mass and \langle\rangle denotes the ensemble average. The knotted ring polymer is allowed to equilibrate for a long time before it is cut randomly at one bond at $t=0$. The nonequilibrium relaxation process is characterized by the time dependence of the radius of gyration $R_{g}(t)$ as it approaches its long time limit. Averages over different realizations (typically $\sim 300-500$ ) of the relaxation processes are performed. Time is measured in units of Monte Carlo steps per monomer (MCS/monomer), one MCS/monomer means that on average every monomer has attempted to move once. 


\section{Knot complexity and equilibrium knot size}

The number of essential crossings $(C)$, i.e., the minimal number of crossings that the knot possesses no matter how one tries to untie it without cutting the string, is the simplest measure of the knot complexity. Quake [10] pictured a knot as a set of interlocked loops with the number of loops determined by the number of essential crossings, predicted the scaling law for the radius of gyration as $R_{g} \propto N^{v} C^{1 / 3-v}$. For good solvents, Flory theory gives $v=\frac{3}{5}$ and $R_{g} \propto N^{3 / 5} C^{-4 / 15}$. Quake has performed Monte Carlo simulations and the result is in agreement with the scaling law. However, $C$ is a fairly weak topological invariant. As we know there are 7 knots with 7 crossings and 166 knots with 10 crossings. The number of knots increases rapidly with the number of crossings. Grosberg et al. [11] recently introduced a new topological invariant $p$ defined as the aspect ratio of the length $(L)$ to the diameter $(d)$ of a knotted polymer at its maximum inflated state, $p=\frac{L}{d} . p$ has a greater value for more complicated knots. It has been demonstrated [15] that $p$ distinguishes rather well between different knot types and thus is a better topological invariant than $C$. For example, the knot $6_{1}(p=29.3)$ is less complex than $6_{3}(p=30.5)$ and the knot $8_{1}(p=37)$ is more complex than $8_{19}(p=31)$. Flory approach has been applied [11] to estimate the equilibrium polymer size by balancing the rubber-like elasticity and volume interactions between monomers and they found that there are four different regimes in which the polymer size has different dependence on $p, N$ and $T$. The four regimes are the good solvent regime, the quasi-Gaussian regime, the poor solvent regime and the maximum tightened knot regime. In the good solvent regime, $R_{g} \sim N^{v} p^{-4 / 15}$. We have performed simulations to verify this relation. In Fig. $2 \mathrm{a},\left\langle R_{g}\right\rangle$ versus $p$ in a log$\log$ plot is shown for chains of various values of $N$. As a reference, $\left\langle R_{g}\right\rangle / N^{v} \simeq 0.38$ for the trivial knot $0_{1}$. Two groups of knots are observed from our data. One group contains the prime knots $\left(3_{1}, 4_{1}, 5_{1}, 5_{2}, 6_{1}, 6_{2}, 6_{3}, 7_{1}, 7_{2}, 8_{1}, 9_{1}\right)$ and the other group consists of the composite knots; $3{ }_{1} \# 3_{1}$ (granny), $3_{1} \#-3_{1}$ (square), $3_{1} \# 4_{1}$, and $3_{1} \# 5_{1}$. Both group show rather good linear relations with slopes relatively close to $-\frac{4}{15}$. However, the values of the radius of gyration for the composite knots are systematically larger than the prime knots for $N=60$ and 82 . This result indicates that the static properties are not only determined entirely by the topological invariant $p$. Also from Fig. 2a and b, we can see that $\left\langle R_{g}\right\rangle$ of $3_{1} \# 3_{1} \# 3_{1}(N=82)$ deviates quite obviously from the linear relation of the group $\left(3_{1} \# 3_{1}, 3_{1} \# 4_{1}, 3_{1} \# 5_{1}\right)$. This further suggests that different number of $f$ actors in the composite knots, such as $3_{1} \# 3_{1}$ and $3{ }_{1} \# 3_{1} \# 3_{1}$, may result in different groups of composite knots. Also it has been recently found [16] that there exists exact additivity of the writhe number but subadditivity of $p$ for the composite knots. They also showed that composite knots with two, three and four prime knots have different degrees of deficits of $p$. Thus, it is plausible to assume that different groups of knots exist. In Fig. 2b, when the $R_{g}$ is rescaled by $N^{v}$, we can see all the data collapse for the prime knots as well as for the composite knots with two factors. This indicates that $R_{g} \sim N^{v}$ is a universal relation. However, it is noted that as $p$ increases, deviation from $R_{g} \sim N^{v} p^{-4 / 15}$ becomes significant. This is because the polymer is crossing 

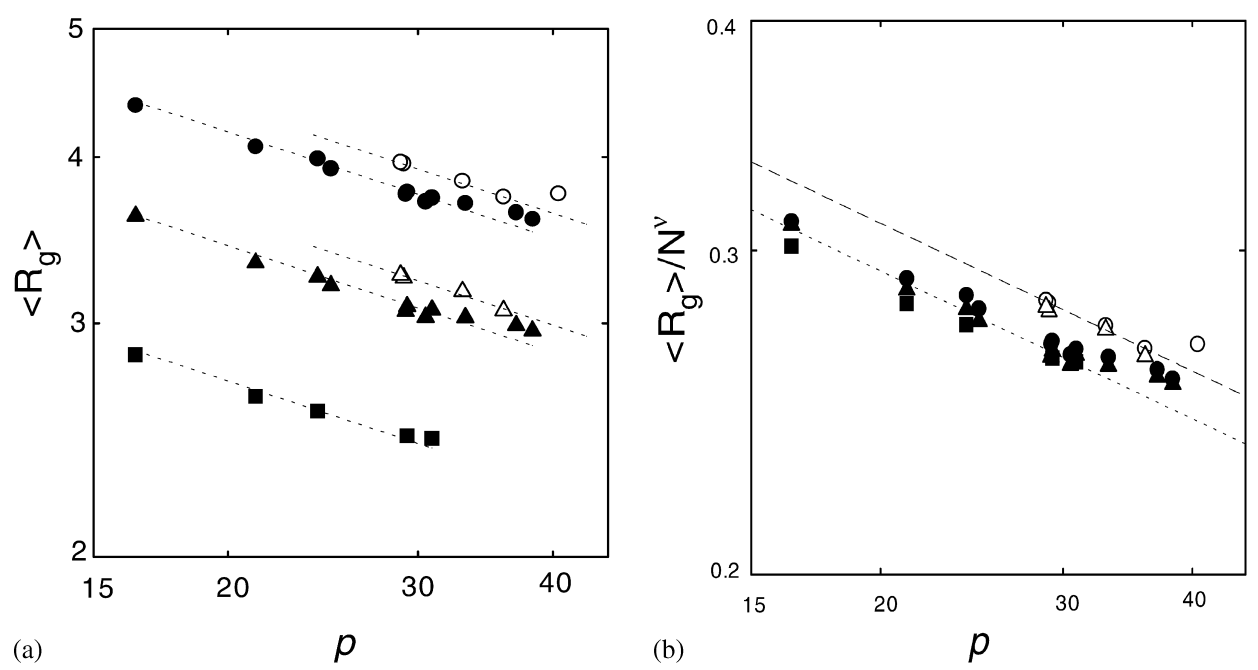

Fig. 2. (a) Radius of gyration $\left(R_{g}\right)$ versus the aspect ratio $(p)$ for knots at various chain lengths. Dotted lines denote slope of $-4 / 15$. Filled symbols represent the prime knots $\left(3_{1}, 4_{1}, 5_{1}, \ldots 9_{1}\right)$ : (ם) $N=42 ;(\boldsymbol{\Delta}) N=60 ;(\bigcirc) N=82$. Open symbols are composite knots: $(\triangle) N=60$ and $(\bigcirc)$

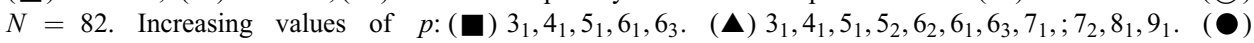

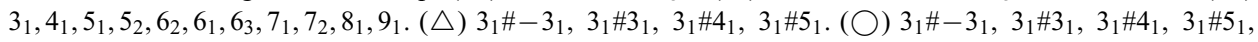
$3_{1} \# 3_{1} \# 3_{1}$. (b) Same data as in (a) but plotted with $R_{g} / N^{v}$ versus $p$.

over to the maximal tightened knot regime. In this regime, the polymer coils up so tightly almost as a compact ball and $R_{g}$ becomes independent of $p$ and solvent quality; $R_{g} \sim N^{1 / 3}$. The uniqueness of this regime is caused by the strong constraint imposed on the knotted ring conformation.

\section{Nonequilibrium relaxation and classification}

Topologically distinct knots, even with the same value of $C$ or complexity, differ from one another in the detail way of tying up the knot. Here we are interested in the question of what physical quantity can best reflect these differences and manifests in some physically measurable quantities. As we have seen in the previous section that equilibrium structural quantities such as the size of the knot, could not resolve very well these differences. Given a knot of a given number of essential crossings or complexity, if one wants to know how it was originally tied up, the simplest way is to cut the knot at some point and untie it to a linear string. Based on this simple idea, but on a molecular level, one can imagine untying the cut knot by Brownian motion and monitor the subsequent nonequilibrium relaxation dynamics. Here we shall focus only on prime knots. The knotted ring polymer is cut at a randomly picked link at $t=0$ and the chain starts to relax towards the Flory coil conformation. $\overline{R_{g}(t)}$ denotes the average of $R_{g}(t)$ over many relaxation realizations and its behavior as a function 


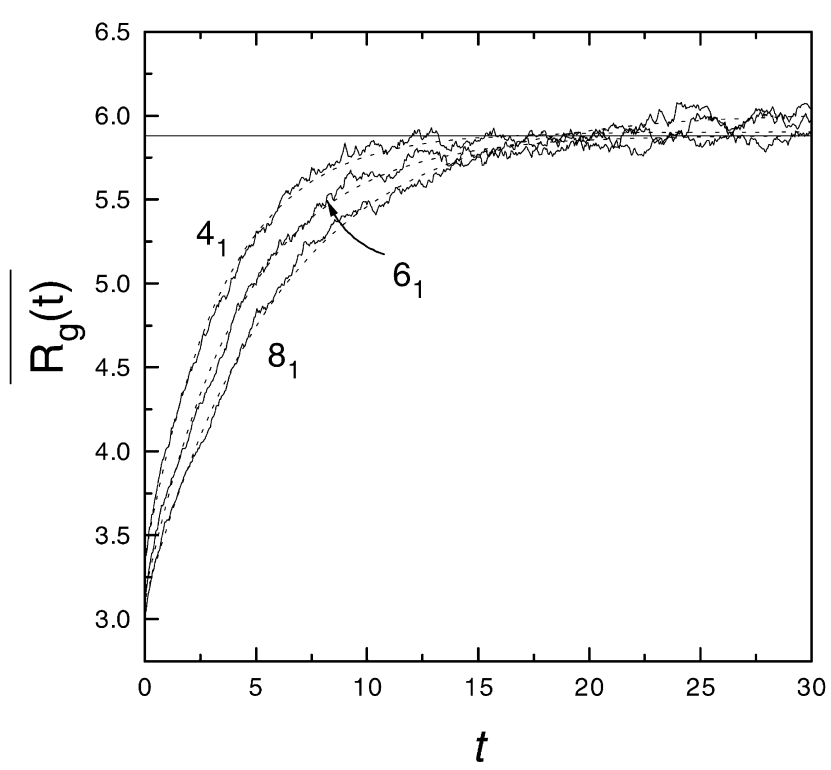

Fig. 3. $\overline{R_{g}(t)}$ versus $t$ for $N=60$ for different knot types. Dotted lines are exponential fits. $t$ is in units of $10^{6} \mathrm{MC}$ steps. The solid horizontal line is the ensemble average $\left\langle R_{g}\right\rangle$ obtained from independent simulations of a linear chain of a chain of the same length at equilibrium.

of $t$ is monitored. Fig. 3 shows the variation of $\overline{R_{g}(t)}$ versus $t$ for $N=60$ with different knot type. As we can see, these chains eventually reach their final equilibrium state of the linear unknotted chain. The relaxation process can be well fitted into an exponential behavior. The nonequilibrium relaxation time $\tau$ is extracted from the data of $\overline{R_{g}(t)}$ by assuming $\overline{\left[R_{g}(\infty)-R_{g}(t)\right] /\left[R_{g}(\infty)-R_{g}(0)\right]}$ decays as $\exp (-t / \tau)$. It is worth noting that this nonequilibrium relaxation differs considerably from the equilibrium correlation time of an uncut knot in which a long time mode exists for the time-autocorrelation function of nontrivial knots [17]. Our results on nonequilibrium relaxations do not show an obvious long time mode and no stretched exponential behavior is observed.

Fig. 4a shows the variation of nonequilibrium $\tau$ versus $p$ for various knots through dynamical Monte Carlo simulations. $\tau$ for the trivial knot $0_{1}$ is also shown for comparison. As we can see, $\tau$ is highly nonmonotonic as $p$ increases. Although the overall trend show, an increase in $\tau$ with increasing $p$, the zig-zag-like local behavior is rather interesting. We believe that the local topological structure plays an even more important role in the relaxation dynamics of a cut knotted polymer as the degree of compactness does. In other words, knots with different detail topological structures relax in different ways. Remarkably, $\tau$ shows a monotonic increasing behavior with $p$ when these prime knots are divided into different groups, based on their topological similarity. These groups are $\left(3_{1}, 5_{1}, 7_{1}, \ldots\right),\left(4_{1}, 6_{1}, 8_{1}, \ldots\right),\left(5_{2}, 7_{2}, 9_{2}, \ldots\right)$, etc. (see also Fig. 1 for these knot groups). Our data indicate the apparently puzzling fact that $3_{1}$ has a longer relaxation time than $4_{1}$ and $6_{1}$, despite that it has fewer crossings and much smaller 


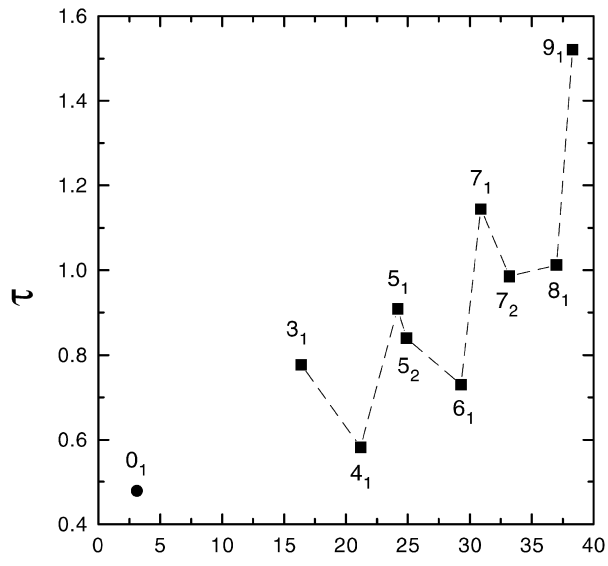

(a)

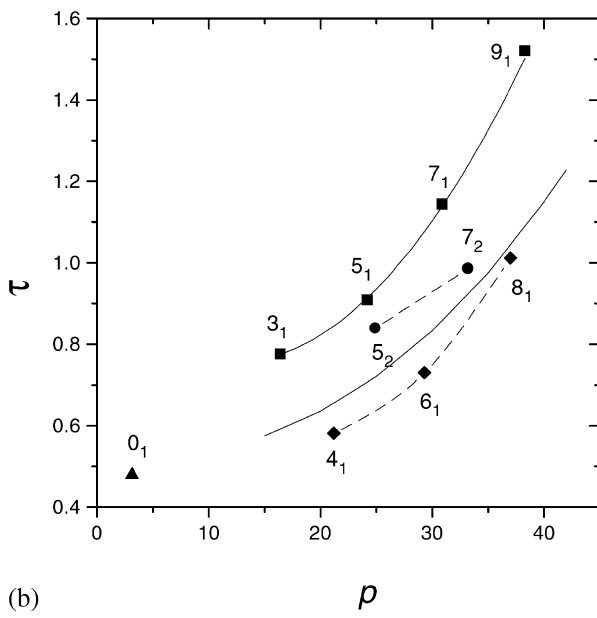

(b)

Fig. 4. (a) Monte Carlo data for the nonequilibrium relaxation time (in units of MCS $/$ monomer $/ 10^{5}$ ) versus $p$ for $N=60$. (b) Same data as in (a) but $\tau$ is separated into different groups. Solid curves are $\tau \sim p^{12 / 5}$.

Table 1

The different knot groups with their Alexander polynomials $\Delta(s)$. $C$ is the number of crossings in the knot. $s$ is just an algebraic variable

\begin{tabular}{ll}
\hline Knot group & Alexander polynomial $\Delta(s)$ \\
\hline$C_{1}:\left(3_{1}, 5_{1}, 7_{1}, \ldots\right)$ & $\left(1+s^{C}\right) /(1+s)$ \\
$C_{1}:\left(4_{1}, 6_{1}, 8_{1}, \ldots\right)$ & $\frac{C}{2}-1-(C-1) s+\left(\frac{C}{2}-1\right) s^{2}$ \\
$C_{2}:\left(5_{2}, 7_{2}, 9_{2}, \ldots\right)$ & $\frac{C-1}{2}-(C-2) s+\frac{C-1}{2} s^{2}$ \\
\hline
\end{tabular}

value of $p$. This can be easily interpreted from our classification since $3_{1}$ belongs to the group of longer relaxation. As from Fig. $4 \mathrm{~b}$ we can see that the group $\left(3_{1}, 5_{1}, 7_{1}, \ldots\right)$ has longer relaxation times than other groups for knots with same chain length. This indicates that the $\left(3_{1}, 5_{1}, 7_{1}, \ldots\right)$ group has the strongest topological hindrance on the relaxation moves among the groups studied. If we plot the relaxation time according to these groups, we find smoothly increasing curves with $\tau \sim p^{12 / 5}$. An important outcome of these results is that the topological effect has a much stronger influence on the nonequilibrium relaxation dynamics than on the equilibrium properties. The radius of gyration for the prime knots and even the equilibrium correlation time of an uncut knot [17], show a consistent monotonic behavior as a function of $p$ while the nonequilibrium relaxation times need to be classified into different groups in order to have a regular monotonic dependence. Furthermore, by analyzing the Alexander polynomials $(\Delta(s))$ of these groups of knots, we find that the knots in each group have a similar form for their Alexander polynomials parametrized by the number of crossing $C$. These polynomials are listed in Table 1 . One can easily see why the relaxation behavior are divided into groups, from the form of the polynomial invariants. The $\left(3_{1}, 5_{1}, 7_{1}, \ldots\right)$ group having 
a long $\tau$ can also be associated with the observation that the degree of $\Delta(s)$ increases with $C$, while for the other two groups their $\Delta(s)$ 's are always of degree 2 .

The importance of the topological effect can be further verified from the outcome of a naive attempt to derive for the relation between $\tau$ and $p$ as follows. If the knot has a greater value of $p$ (i.e., more compact), then simple calculations show that it has a higher free energy difference from the Flory free coil state, and hence the relaxation process proceeds at a faster speed. But this result is contrary to what we have found in simulations. It is because of the existence of the free energy barrier that arose from the topological effect and is not accounted for in the naive free energy difference approach. The system does not simply just relax downhill to the lower free energy Flory coil state, but has to overcome the barrier due to topological constraint of entanglement. Thus for the study of dynamical properties of knots, topological effect is a critical factor. When the chain is cut at $t=0$, the closed ring constraint is relieved and the chain starts to relax. In good solvent regime, the chain would tend to expand out of its compact structure. However, the prohibition against chain crossing in a system hinders the process. Thus, the relaxation process can only proceed through some kind of reptation-like motion through its contour. However, it should be noted that the reptation move is somewhat different from the standard reptation theory. In the reptation theory, cross-linked network or polymer melts are considered and the monomers move in a "tube" resulted from the obstacles produced by other chains. The topology of the surrounding did not change significantly in the intermediate time scales. From our simulation, we find that the radius of gyration expands at the same rate as the end-to-end distance does. In other words, the diameter of the "tube" expands accordingly.

Employing the idea of a maximally inflated tube of contour length $L$ and cross-section diameter $d$, the average time $\tau$ taken by the chain to creep out of the initial contour length $L$ can be evaluated on the basis of the reptation theory as $\tau \sim L^{2} / D$. The diffusion coefficient $D$ can be calculated according to the Einstein relation: $D=k_{B} T / \mu_{t}$ where $k_{B}$ is the Boltzmann constant and $\mu_{t}$ is the total friction coefficient. As we know, the friction coefficient for reptation along a tube is proportional to the number $N$ of links in the macromolecule, i.e., $N \xi$, where $\xi$ is the monomer-solvent friction coefficient in the Rouse model $[18,19]$. However, we believe that an internal friction process is also involved as the chain varies its conformations during the relaxation process. For a linear polymer chain, the monomers tend to avoid each other in good solvents and the probability of two monomers in direct contact is small. However, for knotted polymers, monomers are in close contact because of the existence of crossings. After cutting the knots, in the process of relaxation, monomers will slide onto each other, and extra friction will occur. The collision probability is greatly increased as the number of crossings increases. This monomer-monomer friction does not involve the solvent, but will be somewhat related to the viscosity of a fluid of monomers. We use an analog to electric resistance to estimate this internal friction; that is the monomer-monomer friction coefficient is assumed to be proportional to the ratio of length to cross-section area of the maximally inflated knot. Thus the total friction 


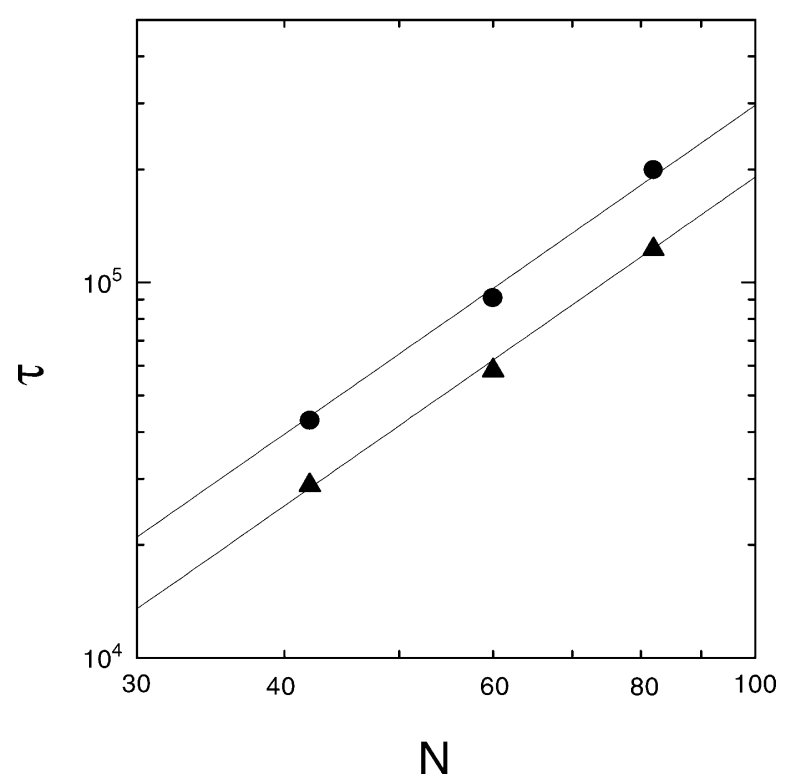

Fig. 5. Nonequilibrium relaxation time $\tau$ (in units of MCS/monomer) versus $N$ for $4_{1}(\boldsymbol{\Delta})$ and $5_{1}(\boldsymbol{O})$ ). Solid lines denote slopes of $1+2 v \simeq 2.2$.

coefficient can be expressed as $\mu_{t}=N \xi+L \zeta$, where $\zeta$ represents the monomer-monomer friction coefficient and $\zeta \simeq \zeta_{o} / d^{2}$ for some characteristic monomer-monomer friction $\zeta_{o}$. Following the idea on the construction of the maximally inflated tube, Grosberg et al. obtained $L \sim R_{g} p^{2 / 3}$ and $d \sim R_{g} p^{-1 / 3} \sim N^{v} p^{-3 / 5}$. Then $\mu_{t}=N \xi+\zeta_{o} N^{-v} p^{8 / 5}$ and hence $\tau \sim L^{2} / D \sim N^{2 v} p^{4 / 5}\left[N \xi+N^{-v} p^{8 / 5} \zeta_{o}\right]$. For fixed $p$ and $N \gg 1, \tau \sim N^{1+2 v}$. For fixed $N$ and $p \gg 1, \tau \sim p^{12 / 5}$. We have performed simulations and plotted $\tau$ against $N$ for $4_{1}$ and $5_{1}$ as shown in Fig. 5. We find that the relation $\tau \sim N^{1+2 v}$ agrees qualitatively well with our simulations. Also, the relation $\left(\tau \sim p^{12 / 5}\right)$ gives good description of our simulation data. However, in the long chain limit $(N \rightarrow \infty)$, one still recovers $\tau \sim N^{1+2 v} p^{4 / 5}$. The cross-over occurs at chain length of $N \simeq p\left(\zeta_{o} / \xi\right)^{5 / 8}$. In the present model in which an attractive square potential exists between monomers, physically we expect $\zeta_{o} \ll \xi$ and the cross-over will occur and chain lengths are much greater than in our present study.

\section{Conclusions and outlook}

The equilibrium structure of uncut knots and nonequilibrium relaxation of cut knotted polymers are studied by Monte Carlo simulations. Our results for the static quantities explicitly verified the scaling laws proposed by Grosberg et al. In the good solvent regimes, the averaged radius of gyration for both the prime and composite knots scale as $R_{g} \sim N^{v} p^{-4 / 15}$ where $p$ is a topological invariant representing the length-to-diameter 
ratio of a knotted polymer at its maximum inflated state. Although $p$ is a better topological invariant than the crossing number $C$, our results for the composite knots indicate that $p$ may not be the determinant invariant in correlating the static properties of all kinds of knots. Simulation results show that the radii of gyration of composite knots are always larger than the prime knots with roughly the same value of $p$. We also found that the dependence of the radius of gyration on $p$ gradually becomes weaker as $p$ increases. We believed that the knotted polymer chain is crossing over to the maximally tightened regime. In the tight knot regime, the polymer is already in extremely compact situation so that an increase of $p$ has no effect on its conformational change.

For the studies of the nonequilibrium dynamics, we monitored the relaxation process of a fully equilibrated prime knotted polymer cut at a randomly picked link. When the constraint of the ring conformation is relieved, the chain starts to relax toward the Flory coil. Especially in good solvent condition, the driving force for the chain to expand is fairly strong. Yet, as we know the self-crossing of the macromolecular sections are forbidden and this effect greatly hinders the relaxation process. As a result, the relaxation proceeds through the reptation-like motions of the chain ends, especially for relatively compact knots. Perhaps the most important finding of this work is that the local topological structure plays a more important role in the relaxation dynamics of a cut knot than $p$ does. The topological effect has much powerful influence on the nonequilibrium relaxation dynamics than on the static or equilibrium properties of knots. The members of each group have similar patterns topologically as can be observed directly by careful visual inspection (see Fig. 1) or from their Alexander polynomials. The members in the $\left(3_{1}, 5_{1}, 7_{1}, \ldots\right)$ group have great resemblance in their knot conformations and their Alexander polynomials can be parametrized by a single formula. Other groups like $\left(4,6_{1}, 8_{1}, \ldots\right)$ and $\left(5_{2}, 7_{2}, 9_{2}, \ldots\right)$ can also be recognized quite easily. The feature of long time mode is especially obvious for $3_{1}$ as compared to $4_{1}, 6_{1}, 8_{1}$. Our results also showed that group $\left(3_{1}, 5_{1}, 7_{1}, \ldots\right)$ has a much longer relaxation time than group $\left(4_{1}, 6_{1}, 8_{1}, \ldots\right)$ does. Somehow, in relaxing its conformation, the group $\left(3_{1}, 5_{1}, 7_{1}, \ldots\right)$ has a topological hindrance that is inherently stronger than other groups. The relaxation time is found to smoothly increase as $\tau \sim p^{12 / 5}$. Naive attempt of estimating the relaxation time from the free energy difference of initial knotted state to the final linear coil state failed indicating the presence of the free energy barrier due to topological effects. On the other hand, the idea of a maximally inflated tube turned out to be useful and analysis based on the reptation theory showed a promising outcome. The average time for a reptating chain to move along its tube by a length $L$ is assumed to be $\tau \sim L^{2} / D$. In addition to the monomer-solvent friction force, the internal friction caused by monomer-monomer drag was also taken into account. The initial ring conformations and the existence of the essential crossings impose very strong constraint on the relaxation path of the chain. Therefore, for knotted chains, monomer-monomer contacts are inevitably frequent. The relaxation time is found to be proportional to $N^{1+2 v}$ for fixed $p$ and $N \gg 1$, which agrees well with our simulation data. For fixed $N$ and $p \gg 1, \tau \sim p^{12 / 5}$ also gives a good description of the data. 
In this work, we focused on studying the relaxation behavior of a cut knotted polymer. Our approach can be readily extended to investigate other properties of knotted chains. For example, Stasiak et al. [12], found that DNA knots can undergo gel electrophoresis and there is a linear relationship between speeds of migration of different types of DNA knots and the average crossing numbers of their ideal geometrical representations. In other words, compact molecules migrate quicker than less compact ones. Until now, there is no consensus about theoretical models that would allow gel migration of a given type of DNA knot to be predicted. We plan to use Monte Carlo methods to study the diffusion rate of knotted polymers in a systematic manner in the hope to help develop and verify the related theories about the mobility of knots. Another interesting issue is how the topological constraints due to knotting a chain molecule would affect the force law or elastic response. There were some recent ab initio calculations [20] on the breaking strength of a polyethlyne chain with a trefoil in it (not a closed chain), but the general physical behavior is not yet revealed and we are currently investigating this problem.

\section{Acknowledgements}

This research is supported by National Council of Science of Taiwan under Grant No. NSC 89-2118-M-008-003. Computing time provided by the Simulational Physics Lab., National Central University is gratefully acknowledged.

\section{References}

[1] W.R. Bauer, F.H.C. Crick, J.H. White, Sci. Am. 243 (1980) 118.

[2] N.R. Cozzarelli, S.J. Spengler, A. Stasiak, Cell 42 (1985) 325.

[3] S.A. Wasserman, N.R. Cozzarelli, Science 232 (1986) 951.

[4] Y. Arai, R. Yasuda, K.-I. Akashi, Y. Harada, H. Miyata, K. Kinoshita Jr., H. Itoh, Nature 399 (1999) 446.

[5] V.F.R. Jones, Bull. Am. Math. Soc. 12 (1985) 103.

[6] G. Burde, H. Zieschang, Knots, Walter de Gruyter, Berlin, 1985.

[7] F.Y. Wu, Rev. Mod. Phys. 64 (1992) 1099.

[8] E. Witten, Commun. Math. Phys. 121 (1989) 351.

[9] L.H. Kauffman, Knots and Physics, 2nd Edition, World Scientific, Singapore, 1993.

[10] S.R. Quake, Phys. Rev. Lett. 73 (1994) 3317.

[11] A.Yu. Grosberg, A. Feigel, Y. Rabin, Phys. Rev. E 54 (1996) 6618.

[12] A. Stasiak, V. Katritch, J. Bednar, D. Michoud, J. Dubochet, Nature 384 (1996) 122.

[13] Y.-J. Sheng, P.-Y. Lai, H.-K. Tsao, Phys. Rev. E 56 (1997) 1900.

[14] M.P. Allen, D.J. Tildesley, Computer Simulations of Liquids, Oxford University Press, New York, 1987.

[15] V. Katritch, J. Bednar, D. Michoud, R.G. Scharein, J. Dubochet, A. Stasiak, Nature 384 (1996) 142.

[16] V. Katritch, W.K. Olson, P. Pieranski, J. Dubochet, A. Stasiak, Nature 388 (1997) 148.

[17] P.Y. Lai, preprint (2000).

[18] P.G. de Gennes, Scaling Concepts in Polymer Physics, Cornell University Press, Ithaca, NY, 1979.

[19] M. Doi, S.F. Edwards, The Theory of Polymer Dynamics, Oxford University Press, Oxford, 1992.

[20] A.M. Saitta, P.D. Soper, E. Wasserman, M.L. Klein, Nature 399 (1999) 46. 